\title{
Contour: A hypermedia environment for teaching about subjective contours and other visual illusions
}

\author{
THOMAS J. DOUGHERTY \\ The Claremont Graduate School, Claremont, California
}

\begin{abstract}
Contour is an interactive Hypercard stack for the Apple Macintosh computer, which presents users with a wealth of information (text, graphics, sound, and animation) concerning the history, theory, and methodology used in the research of a class of visual illusions called subjective contour illusions. The stack is divided into six sections (Introduction, Findings, Index, References, Map, and File Creator), which allow users to access differing types of information in several ways. As users navigate through the stack, they are presented with multimedia information that they can trace through author, theory, or type of illusion. The stack allows users to make notes to themselves on the information presented, to execute a literature search of the most relevant articles in the subjective contour literature, and to save them in text file for printing in a word processing program. The study of subjective contour illusions can be viewed as a microcosm of issues in the field of visual perception.
\end{abstract}

Contour is a courseware Hypercard (Atkinson, 1987) stack designed to teach introductory psychology or perception students about subjective contour illusions. Subjective contours represent a class of optical illusions in which an object, the same color of the background, appears to be partially occluding differently colored objects (see Figure 1). In this figure, the white triangle is partially occluding three black disks. This figure yields three interesting anomalies: (1) illusory brightness, (2) illusory edge, and (3) depth stratification. Simply stated, the figure, in this case a triangle, appears brighter than the background. The triangle also seems to hover above the partially occluded disks and the background. A visible illusory edge can also be seen between the black wedges, where no such edge information exists.

The structure of the Contour stack contains elements of collateral hypertext (Nelson, 1974), which allows users to make simultaneous comparisons between information presented from several different perspectives, and grand hypertext, in which everything about a topic, or pertinent to it, is collected in a single system. In grand hypertext systems, links are presented between different components, creating a loosely structured form. In this way, explanations may be linear or parallel, inductive or deductive, whichever is personally relevant to the user.

Contour is intended to introduce users to this class of visual illusions in an interesting and interactive way. Ex-

The author wishes to thank Glenn E. Meyer for consultation on software content, David Krajicek for comments on initial drafts of this paper, Apple Computer for an equipment grant for this project, and the Alfred P. Sloan Foundation for supplying the hardware with which parts of this program were developed. Correspondence should be addressed to Thomas J. Dougherty, The Claremont Graduate School, Claremont, CA 91711.

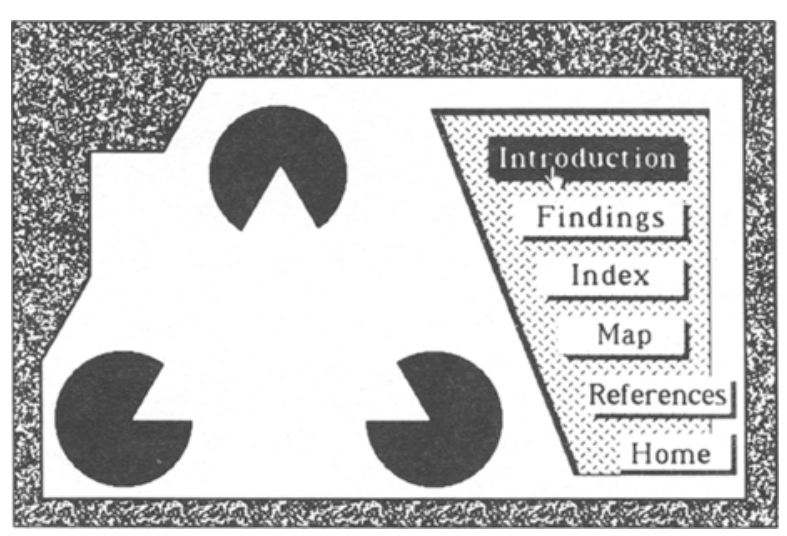

Figure 1. The opening screen with rotating subjective contour.

tensive use of graphics and animation aid in the demonstration of the phenomena and concepts being presented, but also allow the user to be attracted first to the images as a way of peaking interest. Once the user is interested, basic information about the images can be read, and additional information on applicable theoretical and procedural information may be requested, by simply clicking the mouse. The design of the user interface was created to be both inviting and elegant.

The stack consists of six separate sections through which users can navigate. Each section consists of a separate graphic background that best represents the type of information available in that section. The sections are the Introduction screen (see Figure 2), the Findings section (see Figure 3), the Index (see Figure 4), the References section (see Figure 5), the Map (see Figure 6), and the File Creator (see Figure 7). Going from one section of 
the stack to another involves clicking the mouse on buttons that represent the six different sections.

Upon opening the stack, the user is confronted by a rotating animated triangle-that is, a subjective contour figure. It rotates until the user presses the mouse button, at which point several buttons appear (see Figure 1). Here the user may choose from the buttons labeled Introduction, Findings, Index, Map, References, and Home. Clicking buttons on this screen takes the user to the section of his or her choice. The first choice on the list is Introduction, which is a good place for a novice to start.

The Introduction section (see Figure 2) immediately welcomes the user and explains the intent of the stack and the way one can use the navigation buttons to move through the information. There are nine buttons in the navigation window, and the purpose of each button is successively explained in detail. Moving from left to right on the top row of the navigation window are the Index, Note Pad, and Critique buttons. The Index button moves the user to the Index screen, which presents an alternative method of navigating through the information.

Clicking on the Note Pad icon displays a virtual note pad on the screen, covering only the navigation window portion of the screen. Here users can make notes to themselves about the information presented on that card, questions, or comments. Clicking on the close box of the window removes it from the screen, restores the navigation window, and saves the contents of the Note Pad to a hidden field, so that when it is reopened on another card, the previous information remains intact and may then be augmented with additional information. If the user wants to save the contents of the Note Pad, the File Creator section allows the user to organize and edit the notes, and to save them as a text file, which can then be read and printed with any Macintosh word processor.

The Critique button allows the user to request additional information in the form of contradictory findings by other researchers, which are related to the information presented in the text window. This critique field covers the text window, and disappears when it is clicked with the mouse.

The buttons on the second row of the navigation window include Theories, Map, and References. The Theories button, like the Critique button, presents information on the theoretical biases of the research being addressed. An example would be a discussion of brightness theory, if the researcher of the phenomenon being discussed was trying to explain his or her results using brightness theory. The Map and References buttons move the user to the stack Map and the References section, respectively.

On the third row of the navigation window are the Experimental Procedure, Home, and Get Back buttons. Selecting the Experimental Procedure button presents information on the methodology of the study being discussed, in a form analogous to a condensed method section in an APA paper. Clicking the Home icon quits the Contour stack and returns to the Hypercard home card, from which one can quit or launch other stacks. Using the nonlinear structure of hypermedia, one can go from the main Findings section to the References section, for example. After investigating the needed information in the References section, one can use the Get Back button to get the user back to where he or she left off in the Findings section. The Get Back employs the pop-card and push-card statements in Hypertalk, making it possible for the user leaving the fifth card in the Findings section to go to the References section, and then return to the fifth card in the Findings section. It can be used as a hypermedia bookmark.

The Findings section looks like the Introduction section, except that the information here is about the history and research concerning subjective contour illusions, instead of the way to use the interface. For example, Figure 3 shows information on the Ehrenstein illusion, with a historical perspective and other relevant information. Asterisks in the Findings section denote invisible buttons, which are linked to references, and graphics. When

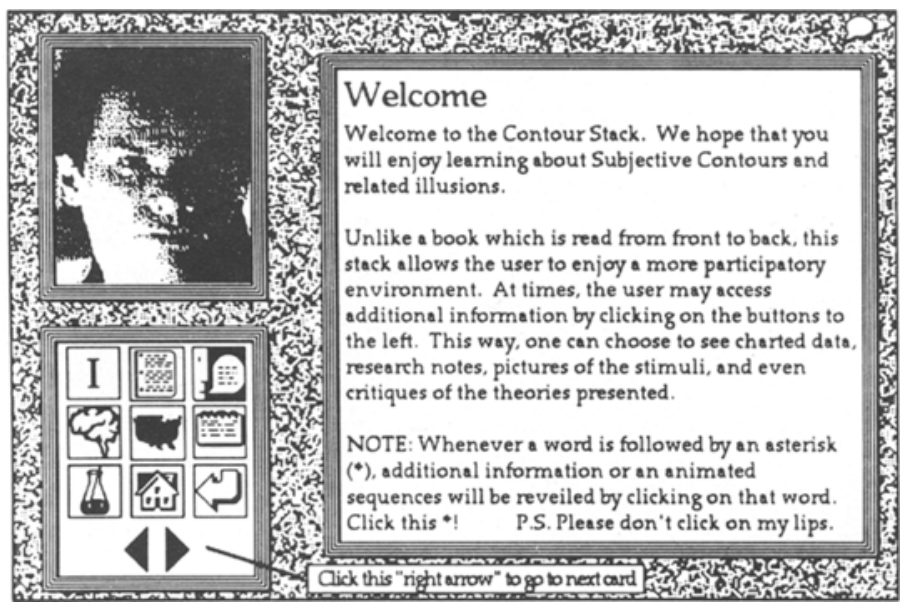

Figure 2. An example of an Introduction screen. 


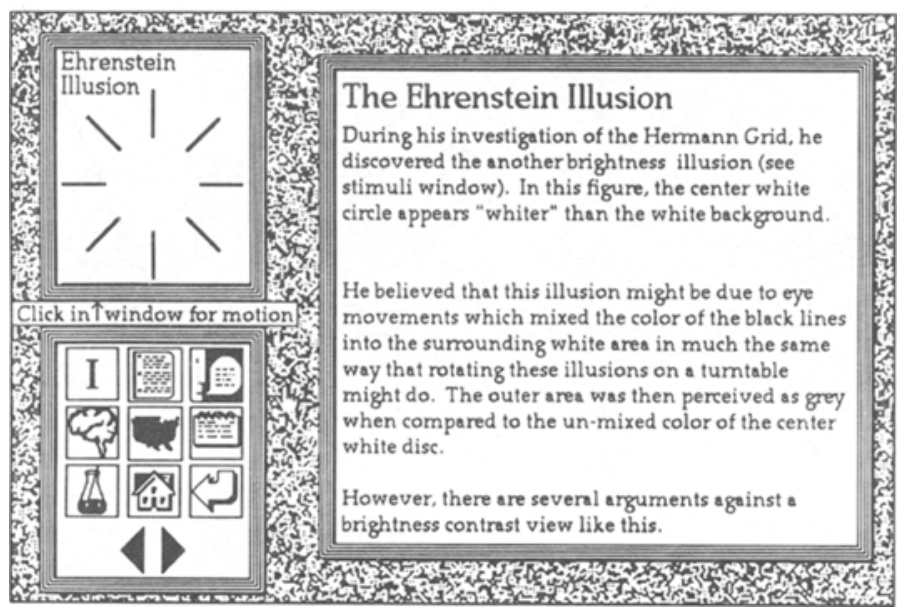

Figure 3. An example of a Findings screen.

a researcher's name is first presented, it is followed by an asterisk. Clicking on that name will present the full bibliographical reference of the research being presented. If one clicks on the image in the Stimulus window, the spokes begin to rotate to illustrate Ehrenstein's explanation of the phenomenon. Selecting the Critique button brings forth evidence that demonstrates why Ehrenstein's explanation was flawed.

The Index card (see Figure 4) allows one to navigate through the stack in a different way. The Index card is essentially a crossroads of buttons. Here items are organized by researcher, theory, and type of illusion. Clicking on any of these buttons moves the user to the appropriate place in the stack. Buttons are also available for going to the References and other sections in the stack.

The References section, designed to look like a stack of note cards on a crumpled piece of typewriter paper, allows each user to execute a literature search of hundreds of the most important articles that relate to subjective contours and other related visual phenomena. The icon on the upper left (see Figure 5) activates a dialogue box that asks for a key word, and for which field (author, title, journal, year, or key words) the user wants to search. By typing a word or name, the user can find citations by certain researchers or on certain topics. Clicking on the Add to Document button on the upper right will restructure the information on this reference card in APA format, and store it in the File Creator section of the stack (see Figure 7).

Selecting the middle button on the right takes one to the File Creator, and allows one to edit both the reference list, and the notes from the note pad. Clicking the Show buttons causes the display to toggle between the reference list and the note pad. Once the information is edited, the user clicks the appropriate buttons to capture this information to an external text file. Opening this text

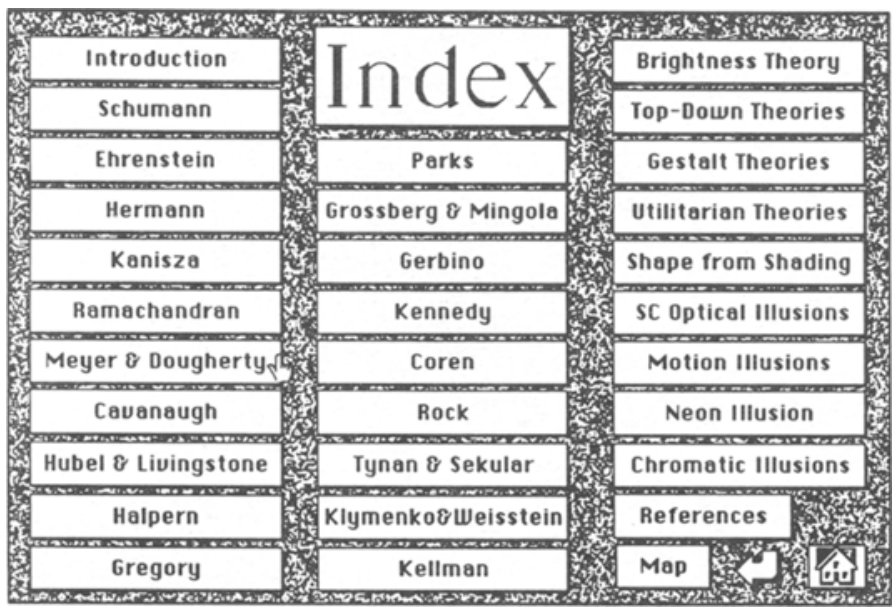

Figure 4. The Index screen. Clicking on any button takes one to information about that researcher or topic. 


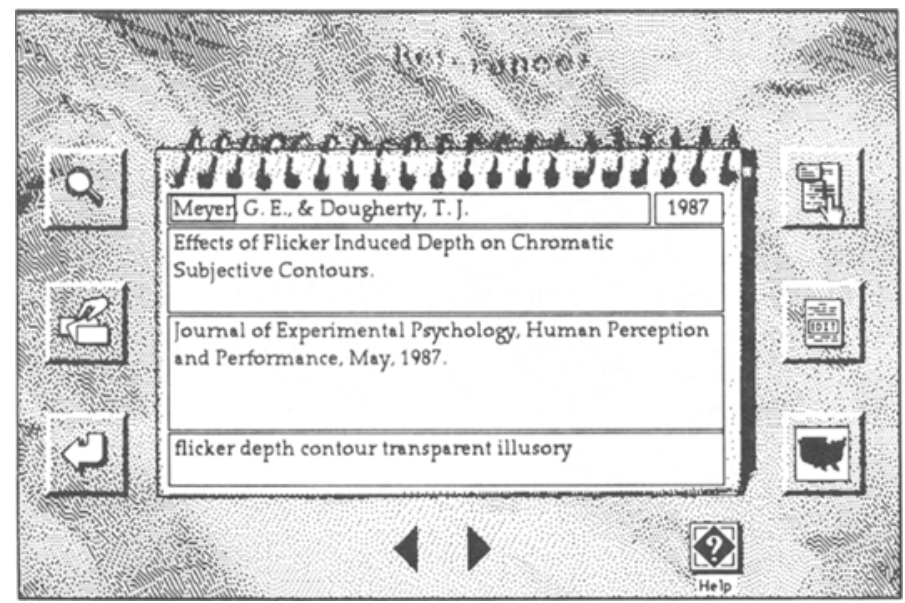

Figure 5. An example of a screen from the References section. Pushing the button at the top right will reformat this reference in APA style and add it to the user's reference list.

file in a word processing program allows the user to print this information, which could be used as a bibliography for a paper on an aspect of subjective contours.

Upon opening, the Map card indicates the stack section from which the user just left by highlighting a facsimile of the background of that section (see Figure 6). In this example, the user had entered the Map card from the Index card. The Map card allows the user to view a schematic of the stack, and, by clicking any of the miniature screens, to go to the corresponding section. This allows the user to create a schema of how the information in the stack is arranged.

\section{Stack Development}

Developing a Hypercard stack can be intimidating, because one can essentially start anywhere. For this reason,
Jonassen's (1986) procedure for developing hypertext environments was helpful. His development chronology includes seven steps: identifying the key concepts, mapping the structure of the content, verifying the structure, determining the type of hypertext structure, preparing concept blocks, defining links, and debugging. Verifying the structure and debugging are important, for they request feedback from others regarding the viability of the concept structure and the intuitiveness of the finished program.

The Contour stack was developed with several programs in addition to Hypercard. Background graphics, like the note card and crumpled paper metaphors in the References section were digitized using MacVision and a video camera. They were saved in MacPaint format and imported into the stack. Some of the stimulus examples were drawn in painting programs such as MacPaint and

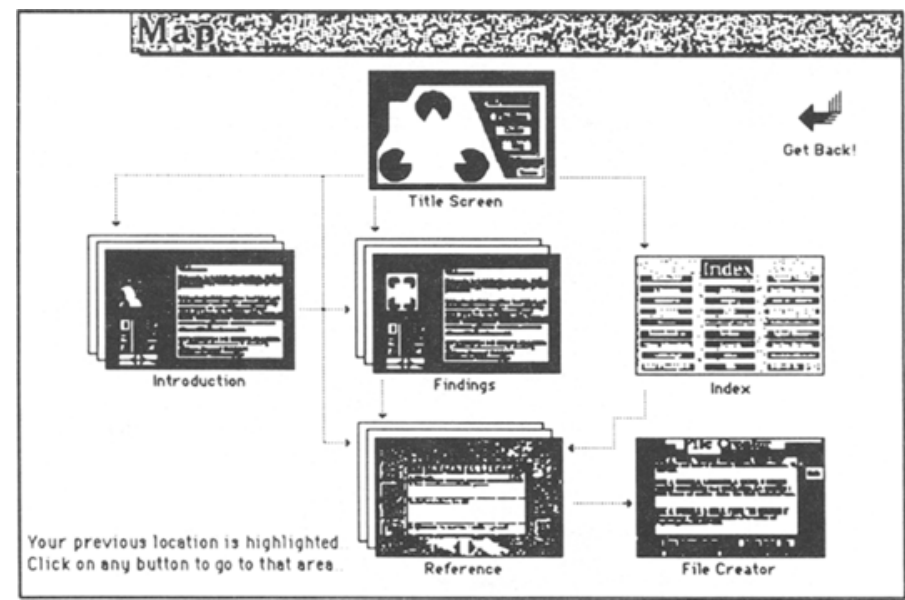

Figure 6. The map of the Contour stack. The highlighted facsimile of the index screen indicates that the user just entered the Map card section of the stack. 


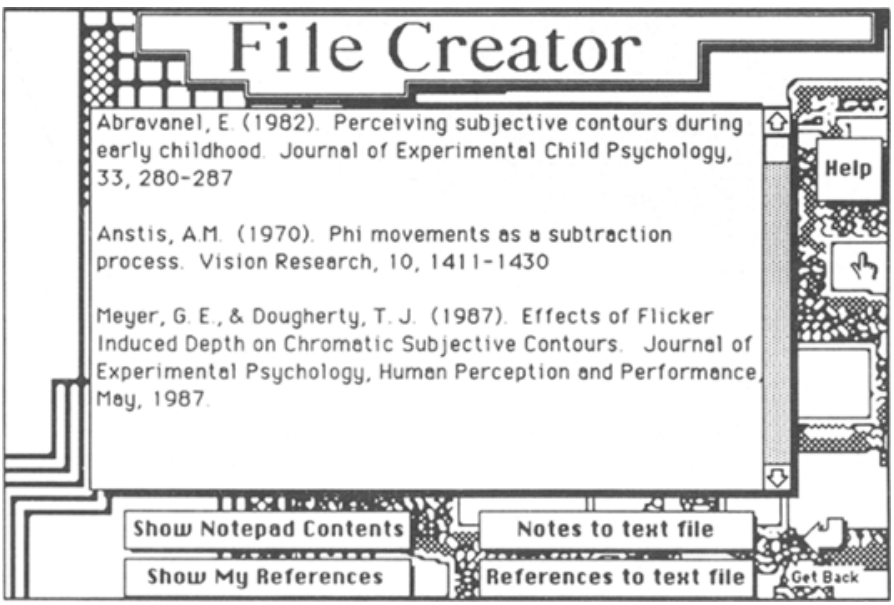

Figure 7. The File Creator card, where the user's references and notes may be edited and saved as text files.

FullPaint, or in object-based graphics programs, such as Cricket Draw and MacDraw II.

\section{System Requirements}

The minimum configuration necessary to use the Contour stack is a Macintosh Plus with 1MB of RAM, and an external $800 \mathrm{~K}$ disk drive. The stack runs much faster, especially with the animated sequences, when one uses a hard disk, but since most college Macintosh laboratories do not have hard drives attached to the computers, every effort was made to keep this software usable on the minimum configured system. Eventually, the addition of colored graphic examples will be made when a critical mass of laboratories are equipped with color Macintoshes.

Animated examples were initially created in Videoworks. Rotating subjective contours were drawn in a graphics program, rotated, and imported to Videoworks. Swivel 3-D was used to recreate the rotating cube illusion by Klymenko and Weisstein. The animated sequences were then assembled, and these files were imported to Videoworks Accelerator, a program that compiles complex Videoworks animations so that they move more quickly and smoothly. These files were then accessed by Hypercard via a Videoworks Accelerator Driver, a licensed XCMD that allows accelerated files to be played within Hypercard stacks.

\section{Program Uses}

The Contour stack is specifically designed for use in courses on introductory psychology and human perception. In introductory psychology, due to the large numbers of students, it is not always possible for an instructor to provide the time and materials necessary to provide active learning-laboratory environments for the perception portion of the course. This is especially true if perception is not the instructor's area of specialty. With hypermedia tools like Contour, the students can work at their own pace, independently, and at their own time, by checking out a disk in the computer lab. The frustration level, for the technology phobic, has been reduced with a very simple, straightforward operating system. One simply points and clicks to get the information one wants. The most basic information is presented on the Findings cards. The more specific information is hidden from view, so that the user will not be intimidated by information he or she chooses not to request.

In perception courses, students may use Contour as an additional lab, to learn more about subjective contours, or to execute a literature search and create a bibliography to help them with their specific term papers on visual illusions. The study of subjective contours is a microcosm of issues in visual perception. In this stack, users are exposed to the concepts of spatial frequency analysis, color perception, top-down and bottom-up processing, anatomy, Gestalt grouping mechanisms, motion and apparent motion perception, and depth perception. This stack can help concretize these abstract concepts, which are often discussed in courses on human perception.

The Contour stack is available directly from the author at $\$ 70.00$ for single copies (institutional site licensing available for $\$ 210.00$ ). A Supercard version, including color phenomena (for Mac IIs), will be available in September 1990.

\section{REFERENCES}

Atxinson, B. (1987). Hypercard [Computer program]. Cupertino, CA: Apple Computer.

JONASSEN, D. H. (1986). Hypertext principles for text and courseware design. Educational Psychologist, 21, 269-292.

Nesson, T. H. (1974). Dream machines. South Bend, IN: The Distributors. 